\title{
Cloud Computing: An Aspect of Information System
}

\author{
Md. Abdullah-Al-Shafi \\ Dept. of Information and Communication \\ Technology \\ Mawlana Bhashani Science and Technology \\ University \\ Tangail, Bangladesh
}

\author{
Ali Newaz Bahar \\ Dept. of Information and Communication \\ Technology \\ Mawlana Bhashani Science and Technology \\ University \\ Tangail, Bangladesh
}

\begin{abstract}
Cloud computing sometimes called on-demand computing is ascending nimble along its data-centre flourishing at a remarkable standard. It appears as a unique and attractive computing pattern that intent to furnish customized, decisive and quality of service guaranteed effective computing situations. Cloud computing offers immense convenience to the IT industry also revamp the IT industry just as revolt although the advancement of this technology is presently at its inception, with many concerns still to be addressed. This paper, an analyzation of cloud computing along its components, architectural basis and challenges of cloud computing also an analysis of mobile cloud computing is presented. The purpose of this paper is to contribute a superior understanding of the architecture challenges of cloud computing.
\end{abstract}

\section{General Terms}

Cloud computing, Information technology.

\section{Keywords}

Cloud computing, Information resources, Information security, Mobile cloud computing.

\section{INTRODUCTION}

Cloud computing exhibits an intrinsic transformation in the form information technology (IT) services are forged, evolved, expanded, amended and provided. New computing follows a paradox in one side, it endures to turn into exponentially more dynamic and per unit amount of computing maintains to fall immediately. Another side, computing comes more prevalent within the system, the rising complication of operating the framework of dissimilar information architectures and shared programs has formed computing further excessive than usual [16]. The new progress of on-demand computing brings a fascinating amount proposition for systems to outsource the IT infrastructure [10] yet expanding interests over the control conceded to massive cloud dealers exclusively the scarcity of data privacy [9] further establishing an ever rising carbon footprint thus increasing environmental interests [11]. Generally [21] four categories of resources perhaps employed over the internet and can be contributed among consumers by leveraging economy of extent. First is infrastructure system like storage, second type is software system encompasses middleware or other resources, third one is application system like Google has serviced cloud platform for association and communication of web utilization [8] and the last one is business procedures. Actually the structure is efficient of service computing like storage devices and self-management [18]. Cloud computing grasps cyber-infrastructure [2], frames grid computing, utility computing and shared computing as well software and web utilities. This paper confers the conception of cloud computing, the architectural design of cloud, the convenience of cloud computing also an analysis of mobile cloud computing is presented.

\section{CLOUD COMPUTING: SYNOPSIS}

Cloud computing is a computing system along the use of the internet for exploring for computational resources where the phrase cloud defines a set of internet resources. The explanation presented by NIST (National Institute of Standards and Technology) [15] emerges to comprise decisive components in on-demand computing: Cloud computing is a model for facilitating conducive, on demand network approach to a shared pool of configurable resources such as servers, storage, networks and applications which can be immediately provisioned and released with nominal management utility provider interaction [15]. Precisely cloud computing system consists of some elemental aspects.

On-demand self-service: A consumer with a spontaneous demand at a distinct moment can avail computing sources, for instance, software use and network storage, without resorting to human communications in an automated manner.

Broad network access: Competence is feasible through the web and accessed over typical systems which improved by thin or thick heterogeneous consumer platform like a mobile phone or laptop.

Resource pooling: A cloud service providers computing resources are pooled to provide various customers using multi-holder fashion along diverse virtual and physical resources effectively appointed and reappointed according to customer's claim [15]. The output of a pool based model is that physical computing resources turn into imperceptible to consumers, who almost do not have control or observation over the location, pattern and originalities resources like CPU.

Rapid elasticity: Effectiveness can be flexibly released and provisioned, in some cases naturally to scale immediately inward and outward commensurate along need. Besides, the provisioning of resource emerges to be enormous to consumers, the consumption can immediately ascent for meet peak concern at any moment.

Measured service: Computing resources are shared and pooled by various customers, the cloud base is adept in using convenient structures measuring the usage of resources. Moreover, the usage can be monitored, reported and maintaining clarity for both the consumer and provider.

\subsection{Architecture of Cloud Computing}

The architecture of cloud computing defines the status of cloud and it can be split into four layers namely application 
layer, the platform layer, infrastructure layer and hardware layer as shown in figure 1.

Application layer: It is the highest level of cloud model which is composed of definite cloud applications. It is different from conventional applications and can leverage automatic scaling factor to obtain superior performance as well reduced performing cost.

Platform layer: Platform layer is the secondary layer that composed of frameworks and operating structure. The function of the platform layer is to diminish the load of expanding application straight into VM storage like App Engine of Google which performs at platform layer to implement API support.
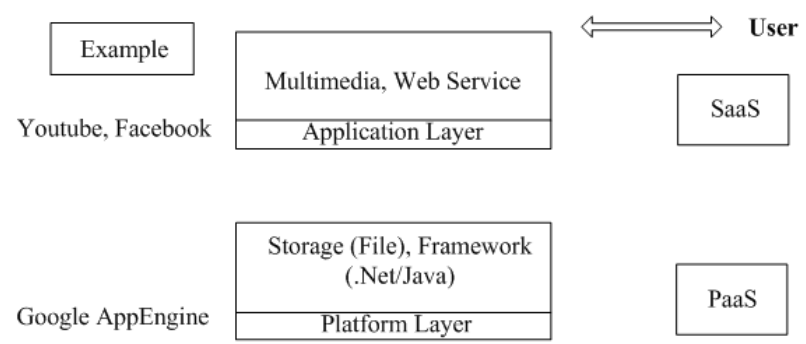

Google AppEngine Platform Laye

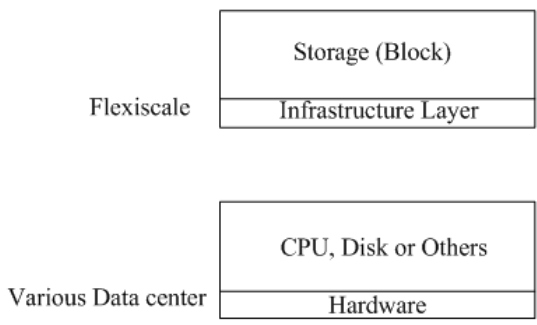

Fig 1: Architectural View of Cloud Computing

Virtualization layer: Also known as infrastructure layer is a crucial fundamental of cloud system as divers vital components like dynamic resource which only made feasible through virtualization technologies. This layer devise computing resources by separating the resources applying virtualization technologies like KVM [14] as well a pool of storage.

Hardware layer: It is the bottom layer of the cloud architecture and liable for maintaining the physical resources of the cloud like routers, cooling systems and power. Basically, this layer contains many servers which interconnected through switches or other devices.

\subsection{Cloud Hierarchy}

In general, the cloud system hierarchy is expansive sets of four universal user levels i.e. users of plain and composite services, domain personnel who coordinate elemental services into composite services and their workflows, authors of underlying operations and various component services, and lastly cyber-infrastructure or (CI) developers as depicts in figure 2 .

User: The most decisive users are end-users of services. User levels comprise indirect users like policy makers as well domain specific groups. An analysis and illustration of this level convenient in the educational domain [20]. The circulation of services across the web as well resources depend on the desired programs and function complication.
Service integration and composition: In this level experts should be capable of focusing on the formation of composite results that is required for an end user. They incorporate and pattern extant services, customize and update services as well inspect usage of the services and assemble service information.

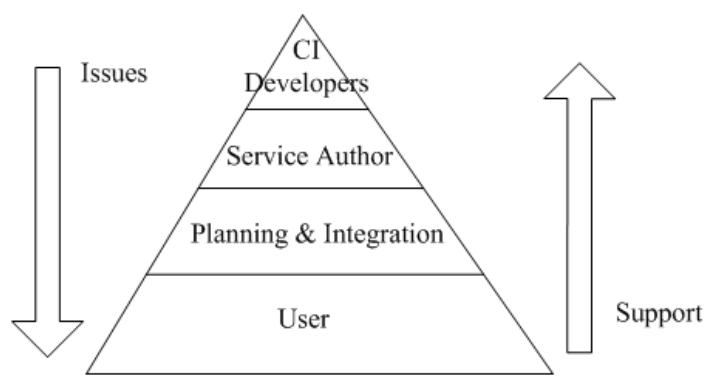

Fig 2: The Hierarchy of Cloud Computing

Service author: Authors are developers of separate baseline services which can be used straight or combined into further complex service aggregates and systems by service provisioning experts. Authors must be component experts as well have positive perceptive of the needs of the user categories shown in Figure 2. Some of the functionalities a cloud structure must implement for them are service registration, license management tools, fault tolerance and others [19].

Developers: Cyber-infrastructure (CI) developers are liable for the development of the cloud structure. They must be experts in functional fields like storage, middleware, networks and so on, also they incorporate system generation, services authoring devices, scheduling algorithms and others.

\subsection{Service Model of Cloud Computing}

The management of cloud is completed centrally. The basic server specifies the balance between customer demand and system capabilities, controls traffic in the network. Users achieve IT infrastructures from clouds system and next run applications inside thus Clouds computing distribute customer along services to approach software, infrastructure and data resources as shown in Figure 3.

Infrastructure as a Service: IaaS specifies to the on-demand provisioning of infrastructural resources. The competence maintained to the user is to provision processing, networks and other elemental resources where a user is capable to expanding and running arbitrary software that encompass applications and operating systems. The proprietor who overtures IaaS is called an IaaS cloud provider like Flexiscale or GoGrid.

Platform as a Service: PaaS specifies to maintaining platform layer resources, including frameworks and software improvement. The capability maintained to the user is to deploy onto the cloud infrastructure user-formed or acquired applications formed using services, programming languages and tools supported by the provider like Google App Engine.

However, in the present tradition, IaaS and PaaS providers are often parts of the same system like Google. That is the reason of IaaS and PaaS providers are usually termed as cloud providers [9]. 


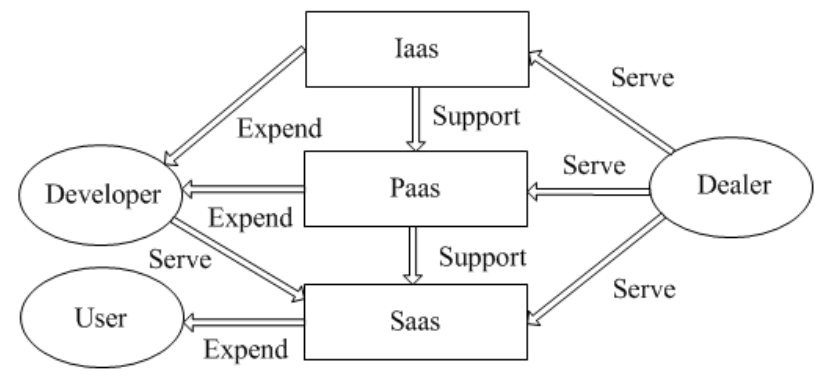

Fig 3: Abstractions of Cloud Computing

Software as a Service: SaaS refers to providing on-demand operations over the internet. The applications are available from different consumer devices through either a thin consumer interface, like the web browser. The user does not maintain the underlying infrastructure including storage, servers or network. An example of SaaS provider is Rackspace.

\section{CATEGORY AND CONVENIENCE OF CLOUD}

Many concerns to acknowledge while shifting an enterprise application to the cloud environment. Therefore, there are various kinds of clouds namely Private cloud, Public cloud, Hybrid cloud and Virtual private cloud.

Private cloud: The cloud infrastructure is managed exclusively in a particular system and maintained by the system or a third party regardless whether it is placed premise or off premise. That is the reason it is recognized as an internal cloud. Internal clouds are formed for exclusive use by a specific system and propose the highest extent of control over authenticity and performance. The reason to setup internal cloud within a system has a distinct aspect like enhancing the utilization of current in-house resources, security matter, data transmission charge [9] from local IT infrastructure and systems regularly need full control over critical movements.

Table 1. Comparison of various representative cloud platforms

\begin{tabular}{|c|c|c|c|}
\hline \multirow{2}{*}{ Feature } & \multicolumn{3}{|c|}{ Organization } \\
\cline { 2 - 4 } & Google & Amazon & Microsoft \\
\hline Focus & Platform & Infrastructure & Infrastructure \\
\hline $\begin{array}{c}\text { Business } \\
\text { Type }\end{array}$ & $\begin{array}{c}\text { Web } \\
\text { application }\end{array}$ & $\begin{array}{c}\text { Storage, } \\
\text { Compute }\end{array}$ & Storage \\
\hline Virtualisation & $\begin{array}{c}\text { Application } \\
\text { container }\end{array}$ & $\begin{array}{c}\text { Xen } \\
\text { hypervisor }\end{array}$ & OS level \\
\hline Web APIs & Yes & Yes & Obscure \\
\hline $\begin{array}{c}\text { Compute } \\
\text { Scheme }\end{array}$ & Python & Linux AMI & $\begin{array}{c}\text { Not } \\
\text { applicable }\end{array}$ \\
\hline
\end{tabular}

Public cloud: This is the main structure of present cloud computing deployment model where providers offer resources to the public. The cloud provider has the complete control of the cloud along its own value, costing, and policy. It offers some basic profits to the providers like changing of risks to infrastructure providers. An example of public cloud is Google App Engine and Amazon EC2.

Hybrid cloud: A hybrid cloud is an infrastructure of private and public cloud models that remain exclusive bodies but are bound together by proprietary technology which facilitates data adjustability. Hybrid clouds are more resilience than both private and public cloud. Systems use the hybrid cloud as improve their resources to raise the basis competencies further it serve compact security over application data contrasted to public clouds.

Virtual private cloud: An explanation to the limitations of both private and public clouds is a virtual private cloud. The primary difference is that a virtual private cloud advantages virtual private network (VPN) technology which permit service providers to form the security context like firewall and individual topology. Sometimes several systems mutually establish and share same infrastructure as well as values and concerns.

On-demand computing offers huge benefits as well as nascent concepts like the resilience of associating business. Especially cloud computing offers some crucial conveniences which are as follows.

Scalability: Computing on cloud forms it effortless for business to scale up or scale down effectively through software APIs depending on consumer [7] and their services that are progressively reliant on exact information in accordance with consumer demand. As the resources are maintained through the program, it is expanded very quickly as recent concerns emerge. Instead of purchasing and installing costly upgrades, the cloud service provider can handle this operation easily.

Immediate access of resources: The refinement of cloud system is that the servers are off-premise. It can implement an immediate connection to hardware resources, with no upfront capital transactions for consumers, leading to a rapid season to market in divers trades [12].

Preservation: On-demand computing provides higher security in business. The information which is saved in the cloud can approach easily no matter what happens to the device also it is possible to erase the information from lost devices so it does not get the wrong person.

Advanced applications and delivers service: Cloud computing generates unique classes of operations and distributes services which were not available earlier. For instances (i) expansions of compute exhaustive applications which offload the data grinding to the cloud leaving alone the distributing of the processed data at the frontend, along the opportunity of connection bandwidth diminishing the latency; (ii) multiple batch processing, which admits consumers to take convenience of large extents of processing influence to evaluate terabytes of data for comparably short periods of time, meantime abstractions like Googles MapReduce [5] or open-source counterpart Hadoop [3] forms complicated process of side by side execution of an application over hundreds of servers translucent to specialists.

Efficient collaboration and globalization: Association in a cloud system provides the capability to share and interact more efficiently outside of the conventional approaches. Cloud computing distributes a protected and swift manner to share financial accounts documents with others. People all over the world can connect cloud provided simply an internet connection.

User-friendly environment: Generally, the cloud provides a friendly environment. If cloud needs to fluctuate the server 
scope scales up or down to fit it. Besides more task is done in a limited period with fewer people.

\section{CHALLENGES OF CLOUD SYSTEM}

Cloud computing is still in its inception so prevailing approval is combined along various challenges. This section deliberates several challenges.

Strategy specification: Scarcity of consolidated strategy specification to cloud system combines proper constraints as well the market specification.

System authenticity: At the current period, various internet user faces the moderate speed to open a website, not to indicate that all the resources are dedicated to the clouds. So what about the loss? Do they have sufficient bandwidth to support the speed?

Contest of the companies: There are several companies in cloud business but still the number is rising. So there's always a competition among the companies. In forthcoming time a colossal problem form that one company shifts to another. How will the system or resources transmit?

Surveillance of data: Still there is no assertion in the cloud system. So what happened if they exchange the information with other users? Will the clouds expose our secrets?

Conservation of security: How will the companies deals with the government in case of secret data of consumers? Sometimes much secret information inserted in the cloud. So how do the company conserve the secrecy?

\section{ANALYSIS ON MOBILE CLOUD COMPUTING}

Mobile cloud computing (MCC) enables the opportunity of cloud computing services in a mobile platform. Basically, mobile cloud computing is a unification of cloud computing, wireless structure or networks, and mobile computing to deliver high performance computational resources to cloud system providers, mobile users, and network operators [1, 13].

Mobile computing provides a new structure for comprising the cloud computing and mobile devices to forge a modern infrastructure where the infrastructure specifies both the data processing and data repository take place outside of the device. The quick evolution of mobile computing (MC) [6] turn into a dynamic flow in the progress of information technology. Still the devices in mobile computing are facing profuse complications in their resources like storage and battery life as well as communications like security and mobility [17]. The following figure depicts the mobile cloud computing.

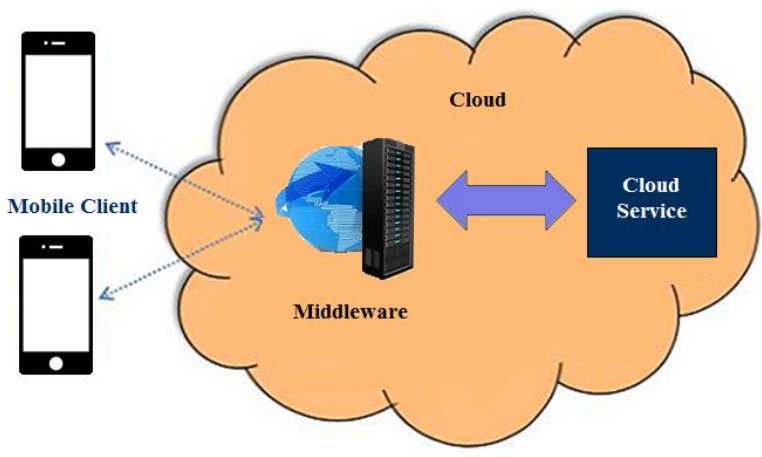

Fig 4: Mobile Computing
Mobile computing has much practical utilization [4] in different areas including image processing, sensor data applications, natural language processing, sharing GPS or internet data, and many more.

\subsection{Characteristic of Mobile Computing}

The primary characteristics of mobile computing are pointed below:

Security: Through the use of various excessive site reliability perhaps enhanced and makes cloud structure more reliable for emergency recovery and business constancy.

Elasticity: Users can quickly connect computing resources beyond human interaction. The Capabilities perhaps immediately provisioned, for some cases dynamically, to quickly extent up or out

Connects wide network: Consumer competence and effectiveness are convenient over the network that can be obtained through standard structures which stimulate use by assorted platforms like the mobile phone or laptop.

Position independence: Position independence is another feature of mobile computing. There is a sense of distinct position independence where user roughly has no control over the definite position of the provided resources. But it can be capable to defining the location at a higher level of abstraction from country, state even data-centre.

\section{CONCLUSION}

Cloud computing has recently appeared as a fascinating model for distributing and managing services through the internet further it frames on decades of exploration in utility and distributed computing as well software and network service. The improvement of on-demand computing is immediately switching information technology, eventually changing the service of computing into a phenomenon. This paper has furnished an overview of cloud computing in which its definitions, components, architecture, challenges, advantages moreover an analysis of mobile cloud computing have been conferred. Overall analysis explains the cloud computing values from the perspective of information resources. Thus, the objective of cloud computing, as well as an illustration of various aspects of cloud computing, is highlighted in the research.

Forthcoming work will focus on Quality of Service (QoS) and Service Level Agreement (SLA) structures in cloud computing with security improvement.

\section{REFERENCES}

[1] Saeid Abolfazli, Zohreh Sanaei, Erfan Ahmed, Abdullah Gani, and Rajkumar Buyya. Cloud-based augmentation for mobile devices: motivation, taxonomies, and open challenges. Communications Surveys \& Tutorials, IEEE, 16(1):337-368, 2014.

[2] Daniel Atkins. Revolutionizing science and engineering through cyberinfrastructure: Report of the national science foundation blue-ribbon advisory panel on cyberinfrastructure. 2003.

[3] Andrzej Bialecki, Michael Cafarella, Doug Cutting, and Owen OMALLEY. Hadoop: a framework for running applications on large clusters built of commodity hardware. Wiki at http://lucene. apache.org/hadoop, 11, 2005. 
[4] Jesse Chang, Rajesh Krishna Balan, and Mahadev Satyanarayanan. Exploiting rich mobile environments. $\mathrm{PhD}$ thesis, thesis, School of Computer Science, Carnegie Mellon University, Pittsburgh, USA, 2005.

[5] Jeffrey Dean and Sanjay Ghemawat. Mapreduce: simplified data processing on large clusters. Communications of the ACM, 51(1):107-113, 2008.

[6] Hoang T Dinh, Chonho Lee, Dusit Niyato, and Ping Wang. A survey of mobile cloud computing: architecture, applications, and approaches. Wireless communications and mobile computing, 13(18):15871611,2013

[7] Abhijit Dubey and Dilip Wagle. Delivering software as a service. The McKinsey Quarterly, 6(2007):2007, 2007.

[8] Google Web Applications for Communication and Collaborations. Google web applications for communication and collaborations. http://www.google.com/apps/. [Online; accessed 02December -2015].

[9] Armando Fox, Rean Griffith, Anthony Joseph, Randy Katz, Andrew Konwinski, Gunho Lee, David Patterson, Ariel Rabkin, and Ion Stoca. Above the clouds: A berkeley view of cloud computing. Dept. Electrical Eng. and Comput. Sciences, University of California, Berkeley, Rep. UCB/EECS, 28:13, 2009.

[10] Mark Haynie. Enterprise cloud services: Deriving business value from cloud computing. Technical report, Micro Focus, Tech. Rep, 2009.

[11] James M Kaplan, William Forrest, and Noah Kindler. Revolutionizing data center energy efficiency. Technical report, Technical report, McKinsey \& Company, 2008.

[12] Won Kim. Cloud computing: Today and tomorrow. Journal of object technology, 8(1):65-72, 2009.
[13] Fangming Liu, Peng Shu, Hai Jin, Linjie Ding, Jie Yu, Di $\mathrm{Niu}$, and Bo Li. Gearing resource-poor mobile devices with powerful clouds: architectures, challenges, and applications. Wireless Communications, IEEE, 20(3):14 $22,2013$.

[14] Kernal Based Virtual Machine. Kernal based virtual machine. http://www.linux-kvm.org/page/Main_ Page/. [Online; accessed 02- December -2015].

[15] Peter Mell and Tim Grance. Draft nist working definition of cloud computing. Referenced on June. 3rd, 15:32, 2009.

[16] Paul Roehrig. New market pressures will drive nextgeneration it services outsourcing. Global Services, $3(35): 22-23,2008$

[17] Philip Tavel. Modeling and simulation design. 2007.

[18] Luis M Vaquero, Luis Rodero-Merino, Juan Caceres, and Maik Lindner. A break in the clouds: towards a cloud definition. ACM SIGCOMM Computer Communication Review, 39(1):50-55, 2008.

[19] Mladen Vouk, Sam Averitt, Michael Bugaev, Andy Kurth, Aaron Peeler, Henry Shaffer, Eric Sills, Sarah Stein, Josh Thompson, et al. Powered by vclusing virtual computing laboratory ( $\mathrm{vcl}$ ) technology to power cloud computing. In Proceedings of the 2nd International Conference on Virtual Computing (ICVCI), pages 15-16. RTP NC, 2008

[20] Mladen Vouk, Donald L Bitzer, Richard L Klevans, et al. Workflow and end-user quality of service issues in webbased education. Knowledge and Data Engineering, IEEE Transactions on, 11(4):673-687, 1999.

[21] Liang-Jie Zhang, Carl K Chang, Ephraim Feig, and Robert Grossman. Business cloud: Bringing the power of soa and cloud computing. In IEEE International Conference on Services Computing (SCC 2008), 2008. 